\title{
Influence of retardation in the scattering of ultracold atoms by conducting nanowires
}

\author{
Martin Fink, ${ }^{1, *}$ Johannes Eiglsperger, ${ }^{2}$ Javier Madroñero, ${ }^{1}$ and Harald Friedrich ${ }^{1}$ \\ ${ }^{1}$ Physik Department, Technische Universität München, D-85747 Garching, Germany \\ ${ }^{2}$ Institut für Theoretische Physik, Universität Regensburg, D-93040 Regensburg, Germany
}

(Received 11 November 2011; published 27 April 2012)

\begin{abstract}
We study low-energy scattering of a neutral atom by a perfectly conducting cylindrical nanowire. Based on the exact atom-wire potential given recently by Eberlein and Zietal [Phys. Rev. A 80, 012504 (2009)] we derive tractable expressions for both the nonretarded van der Waals limit and the highly retarded limit as well as a realistic description of the transition between these two limits. For real atoms and wire radii greater than a few atomic units, the nonretarded van der Waals limit is insufficient, whereas the highly retarded limit of the atom-wire potential gives an accurate description of low-energy elastic scattering and absorption cross sections.
\end{abstract}

DOI: 10.1103/PhysRevA.85.040702

PACS number(s): 34.50.-s, 03.65.Nk, 03.75.-b

A single polarizable atom (or molecule) interacting with an infinitely long, perfectly conducting cylindrical nanowire is a basic example for a hybrid system involving both atomic and nanometer scales. A thorough understanding of this system is a first step toward the understanding of more complex setups involving, e.g., nanogratings that are used in diffraction and interference experiments with atoms or large molecules [1,2]. The atom-wire problem differs from the related problems of an atom interacting with a plane surface or with a sphere in two important features:

(i) Due to translational invariance parallel to the axis of the wire, the system is effectively two-dimensional; scattering theory in two dimensions is somewhat different from the betterknown cases of one or three dimensions and has been receiving increased attention recently [3-5].

(ii) In contrast to the atom-plane and atom-sphere cases, the interaction of an atom with a conducting cylindrical nanowire is rather complicated. Explicit expressions have been given recently by Eberlein and Zietal, both for the nonretarded case [6] and with full inclusion of retardation effects [7].

We recently derived a tractable expression which accurately approximates the nonretarded atom-wire potential as given in [6] and applied it to low-energy atom-wire scattering [8]. In this paper we address the important question of whether retardation effects, which were neglected in [8], have to be considered in the description of atom-wire scattering. We analyze the exact expression for the atom-wire potential as first derived in [7] and confirmed by Bezerra et al. [9]. For the "highly retarded" (HR) limit, in which all transition wavelengths in the atom are assumed to be smaller than the separation of the atom from the surface of the cylinder, we use a procedure analogous to that described in [8] for the nonretarded case and we derive a tractable expression for the atom-wire potential which reproduces the exact potential in the highly retarded limit to any desired accuracy. Based on a full numerical calculation of the interaction between a hydrogen atom and the wire, we show that a phenomenological interpolation between the van der Waals (VDW) limit and the highly retarded limit can reproduce the exact atom-wire potential very well in the

\footnotetext{
*finkm@ph.tum.de
}

intermediate regime between these two limits. We use the resulting atom-wire potentials to study the dependence of atom-wire scattering on the properties of the atom and the radius of the wire. We assume incoming boundary conditions, represented by incoming WKB wave functions, at the surface of the wire. This implies that all atoms which are not reflected by classical or quantum effects in the tail of the atom-wire potential are absorbed, which is a realistic assumption for many scenarios involving the scattering of ultracold atoms by nanostructures. Elastic scattering and absorption cross sections obtained in this way do not depend on details of the atom-wire interaction in the "close" region within a few atomic units of the surface.

The full potential for an atom interacting with a perfectly conducting nanowire involves rather complicated expressions and requires the knowledge of all atomic transition wavelengths [7]. The nonretarded van der Waals limit of the potential is valid when the separation of the atom from the surface of the wire is smaller than all relevant transition wavelengths. The HR limit assumes that the atom-wire separation is larger than all relevant transition wavelengths. In these limits, the potential for an atom of mass $\mu$ can be written as

$$
V^{\mathrm{x}}(r)=-\frac{\hbar^{2}}{2 \mu} \frac{\beta_{\mathrm{x}}^{\alpha_{\mathrm{x}}-2}}{r^{\alpha_{\mathrm{x}}}} \frac{1}{\pi} \sum_{m=-\infty}^{+\infty} \int_{0}^{\infty} d \eta \eta f_{m}^{\mathrm{x}}\left(\frac{r}{R}, \eta\right)
$$

The character $\mathrm{x}$ labels the VDW or HR limits, and the related powers are $\alpha_{\mathrm{VDW}}=3, \alpha_{\mathrm{HR}}=4$. The lengths

$$
\beta_{\mathrm{VDW}}=\frac{2 \mu}{\hbar^{2}} \frac{\left\langle\hat{d}^{2}\right\rangle}{3}, \quad \beta_{\mathrm{HR}}=\sqrt{\frac{2 \mu}{\hbar^{2}} \frac{\alpha_{\mathrm{d}}(0)}{2 \alpha_{\mathrm{fs}}}}
$$

depend on intrinsic properties of the atom: the expectation value of the squared dipole operator $\left\langle\hat{d}^{2}\right\rangle$ and the static dipole polarizability $\alpha_{\mathrm{d}}(0) . \alpha_{\mathrm{fs}}=e^{2} /(\hbar c)$ is the fine-structure constant. Table I lists, as examples, explicit values for hydrogen, helium, and several alkali atoms. Values of $\beta_{\text {VDW }}$ range from a few thousand to several million Bohr radii while values of $\beta_{\mathrm{HR}}$ are in the range of several thousand Bohr radii. 
TABLE I. Explicit values for the expectation value of the squared dipole operator $\left\langle\hat{d}^{2}\right\rangle$, the static dipole polarizability $\alpha_{\mathrm{d}}(0)$ as well as the characteristic length of the atom-wire system in the nonretarded limit $\beta_{\mathrm{VDW}} / 10^{3}$ and in the highly retarded limit $\beta_{\mathrm{HR}} / 10^{3}$, and the characteristic ratio $\rho=\beta_{\mathrm{VDW}} / \beta_{\mathrm{HR}}$.

\begin{tabular}{lccccc}
\hline \hline Atom & $\mathrm{H}[10]$ & $\mathrm{He}[11,12]$ & $\mathrm{He}\left(2{ }^{3} S\right)[13]$ & $\mathrm{Na}[14]$ & $\mathrm{Rb}[14]$ \\
\hline$\left\langle\hat{d}^{2}\right\rangle$ (a.u.) & 3 & 2.256 & 22.8 & 22.68 & 40.32 \\
$\alpha_{d}(0)$ (a.u.) & 4.5 & 1.38 & 316 & 163 & 290 \\
$\beta_{\mathrm{VDW}} / 10^{3}$ (a.u.) & 3.67 & 11.0 & 111 & 633 & 40.68 \\
$\beta_{\mathrm{HR}} / 10^{3}$ (a.u.) & 1.06 & 1.17 & 17.8 & 30.6 & 4190 \\
$\rho$ & 3.45 & 9.33 & 6.24 & 20.7 & 82.4 \\
\hline \hline
\end{tabular}

The functions $f_{m}^{\mathrm{x}}$ indicate the influence of the radius of the wire and are defined in the following way:

$$
\begin{aligned}
f_{m}^{\mathrm{x}}(s, \eta)= & \frac{I_{m}(\eta / s)}{K_{m}(\eta / s)}\left[\eta K_{m}(\eta)\right]^{2} \\
& +\frac{1}{2}\left[\frac{I_{m}(\eta / s)}{K_{m}(\eta / s)}+(-1)^{\alpha_{\mathrm{x}}-1} \frac{I_{m}^{\left(\alpha_{\mathrm{x}}-3\right)}(\eta / s)}{K_{m}^{\left(\alpha_{\mathrm{x}}-3\right)}(\eta / s)}\right] \\
& \times\left\{\left[\eta K_{m}^{\prime}(\eta)\right]^{2}+\left[m K_{m}(\eta)\right]^{2}\right\},
\end{aligned}
$$

where $I_{m}^{\left(\alpha_{\mathrm{x}}-3\right)}$ and $K_{m}^{\left(\alpha_{\mathrm{x}}-3\right)}$ are the derivatives of order $\alpha_{\mathrm{x}}-3$ of the modified Bessel functions $I_{m}$ and $K_{m}$, respectively.

For large radii, both limits reproduce the potential of an atom in front of a plane conducting surface which is proportional to $1 /(r-R)^{3}$ in the van der Waals case and $1 /(r-R)^{4}$ in the highly retarded limit. In the limit of small radii both limits show slowly converging logarithmic series which are not useful for practical purpose [6,8].

A hierarchy of approximations has already been derived in the VDW limit that enables the numerical determination of this potential to any desired accuracy as economically as possible [8]. A similar method can also be used for the HR limit. However, this approach is not applicable for the intermediate region between the nonretarded and highly retarded limit.

In a realistic scenario the interaction potential between an atom and cylindrical wire undergoes a smooth transition from the van der Waals limit at small distances $r \rightarrow R$ to the highly retarded limit at large $r$. Accurate numerical evaluation of the potential is difficult in this transition regime. To describe this regime we generalize the ansatz that has already been used for the potential of an atom in front of a plane surface [15] or a sphere [16]. In these cases, the potential in the transition zone between both asymptotic regimes of the potential was expressed using a shape function $v(x)$ :

$$
V(r)=V^{\mathrm{VDW}}(r)\left\{v\left[V^{\mathrm{VDW}}(r) / V^{\mathrm{HR}}(r)\right]\right\}^{-1},
$$

where $v(x)$ has the following asymptotic properties:

$$
v(x)= \begin{cases}1, & x \rightarrow 0, \\ x, & x \rightarrow \infty .\end{cases}
$$

When the magnitudes of $V_{\mathrm{VDW}}$ and $V_{\mathrm{HR}}$ are very different, $V$ is close to the weaker of the two, which is $V_{\mathrm{VDW}}$ for $r \rightarrow R$ and $V_{\mathrm{HR}}$ for $r \rightarrow \infty$.
For explicit applications of the atom-wire potential in realistic systems, we use two shape functions $v(x)$,

$$
\begin{gathered}
v_{1}(x)=1+x, \\
v_{2}(x)=\frac{\pi / 2}{\arctan [\pi /(2 x)]},
\end{gathered}
$$

which were first introduced by Shimizu [17] and by O'Carroll and Sucher [18], respectively, and have already been applied in similar situations $[15,16]$.

In order to analyze the quality of the approximation (4) we consider a hydrogen atom with the nanowire, which is the only situation where all energy transitions are known exactly. We calculate the potential for a wire with a radius of 1000 a.u. $(\approx 50 \mathrm{~nm})$ and include 1000 transitions to bound states and transitions into the continuum up to an energy of $1000 E_{\mathrm{H}}$, with $E_{\mathrm{H}}=27.2 \mathrm{eV}$, in the exact expression of the atom-wire potential given by Eq. (17) in [7]. The remaining transitions contribute less than $0.001 \%$. The corresponding summation over the index $m$ in the exact atom-wire potential [7] has to be truncated and extrapolated accurately to $m=\infty$. The full circles in Fig. 1 are our results with their respective error bars coming from the extrapolation procedure. The potentials calculated with the help of the shape functions (6) and (7) (dot-dashed and dashed curves, respectively) provide a fair approximation to the exact potential which lies in between.

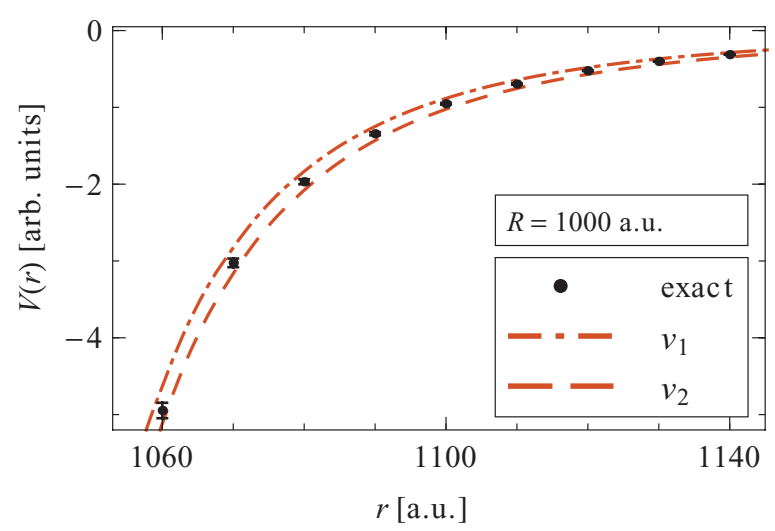

FIG. 1. (Color online) Atom-wire potential (in arbitrary units) for hydrogen atoms and a wire with a radius of 1000 a.u. $(\approx 50 \mathrm{~nm})$. The dot-dashed curve shows the potential approximated using the shape function $v_{1}$ from Eq. (6) and the dashed line shows the approximation using $v_{2}$ from Eq. (7). The black dots show results of an explicit calculation of the atom-wire potential. 
Fundamental properties of the interaction of ultracold atoms with a nanowire can be illustrated by studying the corresponding scattering process. We use incoming boundary conditions to describe the absorption of the projectile at the surface $[15,16]$. The scattering of ultracold atoms is dominated by $s$-wave scattering, and in the regime of low energies the crucial physical quantity is the scattering length which has been defined for two-dimensional systems in $[3,19]$. The dynamics of the scattering process is determined by the radius $R$ of the wire in relation to the characteristic lengths $\beta_{\mathrm{VDW}}$ and $\beta_{\mathrm{HR}}$. Expressing the Schrödinger equation in dimensionless quantities leaves two dimensionless parameters which determine the dynamics, e.g., $R / \beta_{\mathrm{HR}}$ and the characteristic ratio

$$
\rho=\frac{\beta_{\mathrm{VDW}}}{\beta_{\mathrm{HR}}},
$$

the latter being a property of the atom only. Explicit values for $\rho$ are listed in Table I for hydrogen, helium, and several alkali atoms.

The nature of the atom-nanowire interaction can be characterized by the quantity

$$
\xi_{R}(\rho)=\frac{\left|a(\rho)-a_{\mathrm{VDW}}\right|}{\left|a(\rho)-a_{\mathrm{HR}}\right|},
$$

where $a(\rho)$ is the complex scattering length for a given atom with characteristic ratio $\rho$ and $a_{\mathrm{VDW}}\left(a_{\mathrm{HR}}\right)$ is the scattering length in the nonretarded (highly retarded) limit. If the process is dominated by the van der Waals (highly retarded) part of the interaction then $\xi_{R} \ll 1\left(\xi_{R} \gg 1\right)$. Figure 2 shows $\xi_{R}(\rho)$ as a function of the characteristic ratio $\rho$ and the scaled radius of the wire $R / \beta_{\mathrm{HR}}$. The green colored area to the left shows systems where the scattering length has reached its nonretarded limit while the blue colored area to the right shows systems where the scattering length has reached its highly retarded limit. The

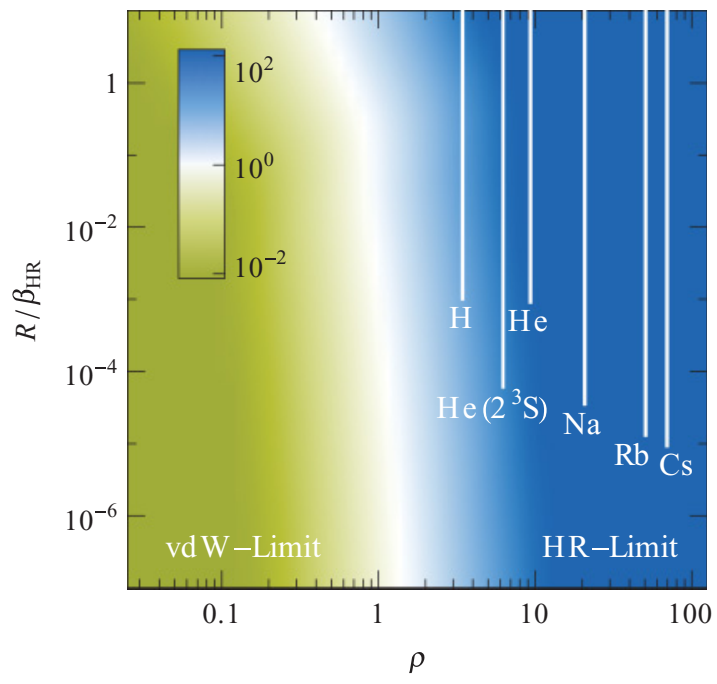

FIG. 2. (Color online) Function $\xi_{R}(\rho)$ on a logarithmic scale as a function of the characteristic ratio $\rho$ and the radius of the wire $R / \beta_{\mathrm{HR}}$. The green colored area to the left shows systems where the scattering length has reached its nonretarded limit $\left(\xi_{R} \ll 1\right)$ while the blue colored area to the right shows systems where the scattering length has reached its highly retarded limit $\left(\xi_{R} \gg 1\right)$. Realistic parameter configurations are shown as white lines. scattering length $a(\rho)$ was calculated using the shape functions (6) and (7) and similar results were obtained in both cases. These were averaged for the calculation of $\xi_{R}$.

As expected, the scattering length undergoes a smooth transition from the nonretarded limit to the highly retarded limit, indicated by the increase of the function $\xi_{R}$ from zero to infinity. This transition takes place around $\rho \approx 1$ and shows only a weak dependence on the radius of the wire. For realistic systems the ratio $R / \beta_{\mathrm{HR}}$ obviously becomes unphysical for $R$ below the Bohr radius. For all atoms listed in Table I, these realistic systems lie in the region where $\xi_{R} \gg 1$ and therefore are dominated by the highly retarded limit of the potential. This is illustrated in Fig. 2, where realistic parameter configurations for these atoms are shown as white lines.

This result is in agreement with what has already been observed for the interaction of a neutral atom and a plane surface. The corresponding potential is reached by the atomwire potential in the limit of large radii, and it has already been shown that in this case the interaction is dominated by the highly retarded part of the potential [15]. Decreasing the radius of the wire has only a weak influence on the transition between the van der Waals and highly retarded limits. For finite and still physically meaningful values of the radius, the system is always dominated by the highly retarded limit. This result is not trivial and is in contrast to what has been observed for an atom in front of an absorbing sphere [16]. In this case, the system can either lie in the highly retarded regime for large radii or, for small but still physically meaningful radii, it can reach the nonretarded van der Waals regime.

To analyze the behavior of the scattering process at higher energies where several partial waves contribute, we study in detail the scattering of hydrogen atoms $(\rho=3.45)$. The scattering process separates into the elastic channel for all atoms that are elastically reflected, due to quantum or classical reflection in the potential tail, and into the absorption channel for all atoms that are absorbed (or inelastically scattered) in the region close to the surface of the wire. We calculated the cross sections for both channels with a fixed nanowire radius of $R=50 \mathrm{~nm}$ as a function of the scaled momentum $k \beta_{\mathrm{HR}}$ up to $k \beta_{\mathrm{HR}}=0.1$, which lies in the $\mathrm{mK}$ region, and with fixed energy corresponding to $T=100 \mu \mathrm{K}$ as a function of the radius up to $R / \beta_{\mathrm{HR}}=10$, for which $R \approx 500 \mathrm{~nm}$. Results for the elastic cross section are shown in Fig. 3(a) as a function of scaled momentum and in Fig. 3(c) as a function of the radius, and results for the absorption cross section are shown in Figs. 3(b) and 3(d). The dot-dashed (dashed) curve shows the results for a potential approximated with the shape function $v_{1}$ from Eq. (6) [ $v_{2}$ from Eq. (7)] and the solid (dotted) curve shows the cross section in the highly retarded (nonretarded) limit.

The figure shows that even at higher energies, both shape functions give results that differ significantly from the VDW limit while only small deviations from the highly retarded limit are observed [in Fig. 3(c) they are almost identical to the HR result and the curves cannot be distinguished]. Changing the projectile atom and going to higher characteristic ratios $\rho$ even decreases these deviations. The difference between the results in the VDW and HR limit decreases with decreasing radius but stays non-negligible for all realistic values, especially for 




FIG. 3. (Color online) Elastic cross section as a function of (a) scaled momentum $k \beta_{\mathrm{HR}}$ and (c) radius $R / \beta_{\mathrm{HR}}$ and (b and d) absorption cross sections for hydrogen atoms $(\rho=3.45)$. The red dot-dashed (dashed) curve shows the cross section for a potential approximated with the shape function $v_{1}$ from Eq. (6) [ $v_{2}$ from Eq. (7)] and the blue solid (green dotted) curve shows the cross section in the highly retarded (nonretarded) limit.

the absorption cross section. Therefore, it becomes clear that for the description of a realistic atom-nanowire system the nonretarded limit is insufficient while accurate results can be obtained from the highly retarded limit.
In a realistic experimental setup, the atom-nanowire system can be realized using a cloud of cold atoms which is brought to full overlap with a nanotube of finite but sufficient length. This guarantees almost perfect translational invariance along the tube axis in the interaction region and therefore realizes the quasi-two-dimensional situation. In this setup, the absorption cross section can be extracted from the atom loss rate which can be measured using state of the art techniques like timeof-flight and absorption imaging methods. This offers a useful possibility to verify our results, in particular as the separation between the nonretarded and the highly retarded limit is larger and therefore easier to resolve than for the elastic cross section. Similar experiments have already been performed to measure loss rates of an ion embedded in a Bose-Einstein condensate or thermal cloud of cold atoms [20,21] or to measure the position of a single carbon nanotube placed on an atom chip [22].

We have presented an accurate description of the interaction of an ultracold atom and a conducting nanowire, based on exact expressions of the atom-cylinder potential both in the nonretarded van der Waals limit and in the highly retarded limit, where all relevant transition wavelengths of the atom are assumed to be smaller than the atom-wire separation. Analyzing the fundamental scattering process we could show that for realistic systems the van der Waals limit is insufficient, whereas the highly retarded limit of the potential provides accurate results.
[1] M. Arndt et al., Nature (London) 401, 680 (1999).

[2] T. Juffmann et al., Phys. Rev. Lett. 103, 263601 (2009).

[3] F. Arnecke, H. Friedrich, and P. Raab, Phys. Rev. A 78, 052711 (2008).

[4] C. Ticknor, Phys. Rev. A 80, 052702 (2009).

[5] N. N. Khuri, A. Martin, J.-M. Richard, and T. T. Wu, J. Math. Phys. 50, 072105 (2009).

[6] C. Eberlein and R. Zietal, Phys. Rev. A 75, 032516 (2007).

[7] C. Eberlein and R. Zietal, Phys. Rev. A 80, 012504 (2009).

[8] M. Fink et al., Phys. Rev. A 81, 062714 (2010).

[9] V. B. Bezerra et al., Eur. Phys. J. C 71, 1614 (2011).

[10] H. A. Bethe and E. E. Salpeter, in Handbuch der Physik, Vol. XXXV, edited by S. Flügge (Springer-Verlag, Berlin, 1957).

[11] Z.-C. Yan, J. F. Babb, A. Dalgarno, and G. W. F. Drake, Phys. Rev. A 54, 2824 (1996).

[12] C. Pekeris, Phys. Rev. 115, 1216 (1959).
[13] Z.-C. Yan and J. F. Babb, Phys. Rev. A 58, 1247 (1998).

[14] A. Derevianko, W. R. Johnson, M. S. Safronova, and J. F. Babb, Phys. Rev. Lett. 82, 3589 (1999).

[15] H. Friedrich, G. Jacoby, and C. G. Meister, Phys. Rev. A 65, 032902 (2002).

[16] F. Arnecke, H. Friedrich, and J. Madroñero, Phys. Rev. A 75, 042903 (2007).

[17] F. Shimizu, Phys. Rev. Lett. 86, 987 (2001).

[18] M. O'Carroll and J. Sucher, Phys. Rev. 187, 85 (1969).

[19] B. Verhaar, J. P. H. W. van den Eijnde, M. A. Voermans, and M. M. J. Schaffrath, J. Phys. A 17, 595 (1984).

[20] C. Zipkes, S. Palzer, C. Sias, and M. Köhl, Nature (London) 464, 388 (2010).

[21] S. Schmid, A. Härter, and J. H. Denschlag, Phys. Rev. Lett. 105, 133202 (2010).

[22] M. Gierling et al., Nature Nanotechnology 6, 446 (2011). 